\title{
Soluble P-selectin promotes acute myocardial infarction onset but not severity
}

\author{
LING GUO ${ }^{1}$, GUIZHI SUN ${ }^{1}$, GUOYU WANG ${ }^{2}$, WENHU NING $^{3}$ and KAN ZHAO $^{3}$ \\ Departments of ${ }^{1}$ Cardiology, ${ }^{2}$ Pharmacy and ${ }^{3}$ Emergency, The Fourth Affiliated Hospital of Harbin Medical University, \\ Harbin, Heilongjiang 150001, P.R. China
}

Received March 20, 2014; Accepted August 19, 2014

DOI: $10.3892 / \mathrm{mmr} .2014 .2917$

\begin{abstract}
P-selectin, an integral membrane glycoprotein of platelets and endothelial cells, and the soluble form of P-selectin are hypothesized to play a role in the initiation of atherosclerosis and acute myocardial infarction (AMI). However, limited data are available with which to evaluate the main role of soluble P-selectin (sP-selectin) in the onset or the severity of AMI. In the present study, we investigated 15 patients who suffered from angina, 10 patients who underwent percutaneous coronary intervention (PCI) therapy and 10 patients who underwent thrombolysis therapy, compared with 15 volunteers with no cardiovascular disease. We confirmed that the plasma sP-selectin levels were increased in patients with obesity (particularly pericardial obesity) and hyperlipidemia, positively correlated with plasma tumor necrosis factor (TNF)- $\alpha$ and strongly negatively correlated with adiponectin in all patients regardless of AMI status. Furthermore, sP-selectin levels were significantly higher in PCI and thrombolysis patients compared with angina patients and the control cohort. However, we observed that sP-selectin levels did not change following PCI and thrombolysis therapy. In addition, there was no correlation between sP-selectin levels and the severity of AMI in the cohort which received PCI or thrombolysis therapy. Therefore, we deduced that sP-selectin only induced the onset of AMI but did not promote its severity. To confirm this hypothesis, a P-selectin inhibitor was administered to an atherosclerosis formation model, plaque rapture model and neointimal hyperplasia model. We revealed that atherosclerotic plaque formation and rupture, neointimal formation and neointimal bleeding were suppressed by the sP-selectin inhibitor. We concluded that sP-selectin, induced by systemic inflammation in conditions including obesity
\end{abstract}

Correspondence to: Professor Kan Zhao, Department of Emergency, The Fourth Affiliated Hospital of Harbin Medical University, 37 Yiyuan Street, Nangang, Harbin, Heilongjiang 150001, P.R. China

E-mail: zhaokan451@gmail.com

Key words: (soluble) P-selectin, acute myocardial infarction, atherosclerotic plaque, plaque rupture, neointimal formation and hyperlipidemia, promoted atherosclerotic plaque and neointimal formation, plaque rapture and neointimal bleeding, further leading to AMI. We also demonstrated that sP-selectin had no effect on the severity of AMI.

\section{Introduction}

P-selectin (CD62P) is a member of the selectin family of cell adhesion molecules located in the platelet granule and Weibel-Palade body of endothelial cells. P-selectin mediates the rolling of blood cells on the surface of the endothelium and initiates the attachment of leukocytes circulating in the blood to platelets, endothelial cells and other leukocytes at sites of tissue injury and inflammation (1). These processes are possible since P-selectin glycoprotein ligand-1 (PSGL-1), the ligand for P-selectin, is present on the surface of platelets and endothelial cells which undergo sulfation of a specific tyrosine residue at the $\mathrm{N}$-terminal for P-selectin recognition $(2,3)$. Soluble P-selectin, lacking the cytosolic/transmembrane domain, has been identified as circulating in plasma in healthy controls and diseased cohorts. An increased level of soluble P-selectin (sP-selectin) is a major predictive factor of cardiovascular events relating to platelet turnover and its activation and function. P-selectin plays a key role in diseases associated with injury and arterial thrombosis. Increased expression of P-selectin is observed in coronary artery disease, acute myocardial infarction (AMI), stroke and peripheral artery diseases $(4,5)$.

Inflammation plays a significant role in numerous chronic diseases, including atherosclerosis-associated cardiovascular disease. The adhesion of immune cells plays a critical role in the inflammatory response and indeed the pathophysiology of inflammatory diseases. P-selectin is critical in the progression of atherosclerosis as evidenced by knockout animal models, where P-selectin knockout mice crossed with ApoE-deficient mice exhibited significantly reduced atherosclerosis and leukocyte recruitment in the plaque. $\mathrm{sP}$-selectin also has pro-atherogenic and pro-thrombotic effects [as reviewed in (6)]. More than $80 \%$ of acute myocardial infarcts are the result of coronary atherosclerosis with superimposed luminal thrombus and its rupture. Uncommon causes of myocardial infarction include coronary spasm, coronary embolism and thrombosis in nonatherosclerotic normal vessels (7). These findings led us to hypothesize that sP-selectin-associated AMI is induced by coronary atherosclerosis formation and rupture. 
As the primary adhesion molecule in initiating cell activation and cell adhesion to platelets and endothelial cells, and having a critical role in inducing chronic inflammatory and cardiovascular diseases, $\mathrm{P}$-selectin is an attractive therapeutic target in vascular diseases. However, the basic tenet of targeting P-selectin may be complicated by the presence of a soluble form of P-selectin; thus, the targeting of P-selectin remains a strong clinical candidate for developing novel therapeutic strategies (8). Percutaneous coronary intervention (PCI) and thrombolysis are the two main therapeutic strategies for AMI (9). Since the two strategies are used to remove the obstruction of the coronary artery to prevent AMI, there is a possibility that PCI or thrombolysis could inhibit sP-selectin to a certain extent. sP-selectin is increased in AMI and atherosclerosis patients, which proves only that sP-selectin is correlated with the onset of AMI. However, it remains unclear whether sP-selectin also affects the severity of AMI.

In the present study, we investigated P-selectin in 50 patients and the role of $\mathrm{sP}$-selectin by administration of P-selectin antagonist in two mouse models (atherosclerosis model and neointimal formation model). We demonstrated that $\mathrm{sP}$-selectin only promoted atherosclerotic plaque and its rupture, and neointimal formation and bleeding, then induced the onset of AMI but did not affect its severity.

\section{Materials and methods}

Human sample collection. For human analysis, baseline plasma samples were obtained from 50 male cohorts from the Fourth Affiliated Hospital of Harbin Medical University (Harbin, China). Ages ranged from 42-67 years. These comprised 15 control cohorts, who had no any cardiovascular disease; 15 chest pain cohorts who were diagnosed with angina; 10 cohorts who were diagnosed with AMI and finally underwent PCI therapy; and 10 cohorts who were diagnosed with AMI and finally underwent thrombolysis therapy. Written informed consent was obtained from the patients. For the 20 AMI cohorts, the therapeutic choice of PCI or thrombolysis therapy was voluntary. None of the individuals involved in this experiment suffered from any $\mathrm{sP}$-selectin-induced disease, such as chronic obstructive pulmonary disease, rheumatoid arthritis and dermatitis, in which sP-selectin is reportedly increased (10-12).

Human background and plasma measurement. For all cohorts, the body mass index (BMI) and waist circumference (WC) were measured as a routine examination. Pericardial adipose tissue (PCAT) volume was measured using 64-slice computed tomography and then calculated three-dimensionally using contrast-enhanced images, as reported previously (9).

Plasma sP-selectin, total cholesterol (TC), low-density lipoprotein (LDL), high-density lipoprotein (HDL), tumor necrosis factor (TNF)- $\alpha$, adiponectin, creatine kinase (CK)-MB and cardiac troponin I (cTnl) were measured by a specific ELISA kit (MyBioSource, Inc., San Diego, CA, USA). The basic data are shown in Table I.

Animal models. To generate the atherosclerotic formation and plaque rupture models, male $A p o E^{-/-}$mice (Biocytogen Co.,
Ltd., Beijing, China), aged 12 weeks, were fed a high-cholesterol diet for 5 and 10 weeks $(n=12$ each), as described previously $(13,14)$.

To generate the neointimal hyperplasia and neointimal bleeding model, the bilateral femoral arteries of 16-week-old C57BL/6N mice were subject to transluminal wire injury as described previously (15). The neointimal hyperplasia mice were fed a normal diet and the neointimal bleeding mice were fed a high-cholesterol diet for 5 weeks. All mice had ad libitum access to water. The present study was approved by the ethics committee of Harbin Medical University.

Antibody administration. In the atherosclerotic group, each mouse was given a single bolus of $100 \mu \mathrm{g}$ RB40.34 mAb (Thermo Fisher Scientific Inc., Waltham, MA, USA) via IP injection once a week for 5 weeks.

In the neointimal hyperplasia group, three hours before femoral arterial injury, each mouse was given a single bolus of $100 \mu \mathrm{g}$ RB40.34 mAb via IP injection with the operator blinded to treatment (16).

Tissue staining and the evaluation of plaque and neointimal hyperplasia. Anesthetized mice were perfused with phosphate-buffered saline (PBS) through the left cardiac ventricle, and then tissues were fixed by perfusion of $4 \%$ paraformaldehyde containing PBS. For neutral lipid visualization, sections were rinsed in $60 \%$ isopropanol and incubated in a saturated, filtered solution of Oil Red $\mathrm{O}$ in $60 \%$ isopropanol for $1 \mathrm{~h}$. Elastica van Gieson staining was performed as reported previously (17).

Statistical analysis. Data are expressed as the means \pm standard error of the mean of triplicate runs. Each experiment was repeated at least three times. Student's t-test and analysis of variance were used to assess differences, and $\mathrm{P}<0.05$ was considered to indicate a statistically significant difference.

\section{Results}

sP-selectin is increased in obesity and hyperlipidemia patients. Since sP-selectin, which induces the adhesion of platelet and endothelium cells, is reported to be an inflammatory factor, and obesity is a well-known cause of classic chronic systemic inflammation, the BMI and WC indices are often used to evaluate obesity (18). Therefore, we firstly investigated the correlation of sP-selectin and obesity using these two obesity indices in all 50 patients, regardless of their AMI status. sP-selectin levels were found to be $90 \pm 14$, $128 \pm 35$ and $57 \pm 54 \mathrm{ng} / \mathrm{ml}$, respectively, in the three BMI subgroups $(\mathrm{BMI}<25,25<\mathrm{BMI}<30$ and $\mathrm{BMI}>30$; Fig. $1 \mathrm{~A})$, in which 25 and 30 were excluded, and sP-selectin levels were $108 \pm 27$ and $158 \pm 59 \mathrm{ng} / \mathrm{ml}$, respectively, in the two WC subgroups ( $\mathrm{WC}<80 \mathrm{~cm}$ and $\mathrm{WC}>80 \mathrm{~cm}$; Fig. 1B; excluding $80 \mathrm{~cm}$ ). In previous studies, PCAT was reported to have a higher correlation with coronary heart disease than abdominal obesity $(13,20)$. We therefore supposed that sP-selectin was also correlated with PCAT. It was demonstrated that sP-selectin was $148 \pm 55 \mathrm{ng} / \mathrm{ml}$ in PCAT areas over $160 \mathrm{~cm}^{2}$, which was higher than the $110 \pm 41 \mathrm{ng} / \mathrm{ml}$ measured in areas less than $160 \mathrm{~cm}^{2}$ (Fig. 1C) $160 \mathrm{~cm}^{2}$ itself was not assessed. 
Table I. Characteristics of patients.

\begin{tabular}{lcccc}
\hline & $\begin{array}{c}\text { Control } \\
(\mathrm{n}=15)\end{array}$ & $\begin{array}{c}\text { Chest pain } \\
(\mathrm{n}=15)\end{array}$ & $\begin{array}{c}\text { PCI } \\
(\mathrm{n}=10)\end{array}$ & $\begin{array}{c}\text { Thrombolysis } \\
(\mathrm{n}=10)\end{array}$ \\
\hline Gender & Male & Male & Male & Male \\
Age & $49 \pm 7$ & $52 \pm 8$ & $55 \pm 12$ & $56 \pm 9$ \\
sP-selectin $(\mathrm{ng} / \mathrm{ml})$ & $101 \pm 20$ & $120 \pm 29$ & $156 \pm 57$ & $166 \pm 76$ \\
BMI & $24.4 \pm 3.4$ & $26.7 \pm 3.6$ & $30.2 \pm 4.0$ & $28.1 \pm 3.6$ \\
WC $(\mathrm{cm})$ & $80 \pm 9.7$ & $81 \pm 8.5$ & $92.3 \pm 9.3$ & $97.1 \pm 8.5$ \\
PCAT & $154 \pm 14$ & $162 \pm 16$ & $169 \pm 16$ & $162 \pm 17$ \\
TC $(\mathrm{mmol} / \mathrm{l})$ & $4.02 \pm 0.66$ & $4.39 \pm 0.73$ & $3.29 \pm 1.30$ & $5.62 \pm 1.51$ \\
LDL $(\mathrm{mmol} / \mathrm{l})$ & $2.14 \pm 0.64$ & $2.98 \pm 0.77$ & $1.07 \pm 0.24$ & $3.43 \pm 1.25$ \\
HDL $(\mathrm{mmol} / \mathrm{l})$ & $1.51 \pm 0.28$ & $1.15 \pm 0.39$ & $1.02 \pm 0.38$ & $1.15 \pm 0.34$ \\
TNF- $\alpha(\mathrm{ng} / \mathrm{ml})$ & $0.83 \pm 0.41$ & $0.73 \pm 0.35$ & $5.89 \pm 1.67$ & $1.22 \pm 0.39$ \\
Adiponectin $(\mu \mathrm{g} / \mathrm{ml})$ & $7.06 \pm 1.77$ & $7.39 \pm 1.5$ & $54.8 \pm 14.4$ & $6.52 \pm 2.45$ \\
CK-MB $(\mathrm{U} / \mathrm{l})$ & - & - & $2.23 \pm 1.08$ & $44.2 \pm 13.8$ \\
cTnl $(\mu \mathrm{g} / \mathrm{l})$ & - & - & $2.88 \pm 0.75$ \\
\hline PCl & & & & \\
\hline
\end{tabular}

PCI, percutaneous coronary intervention; BMI, body mass index; WC, waist circumference; PCAT, pericardial adipose tissue; TC, total cholesterol; LDL, low-density lipoprotein; HDL, high-density lipoprotein; TNF- $\alpha$, tumor necrosis factor- $\alpha$; CK-MB, creatine kinase-MB; cTnI, cardiac troponin I.

A

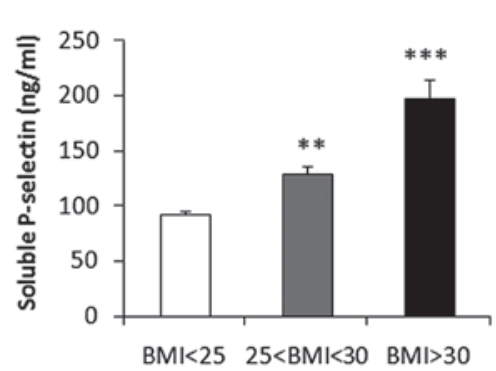

C

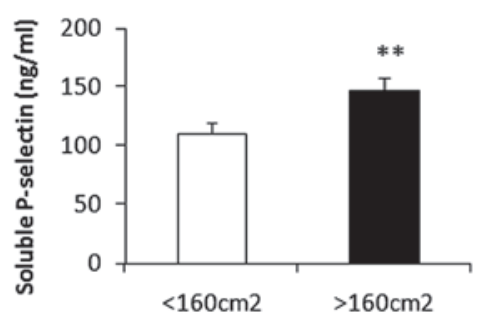

E

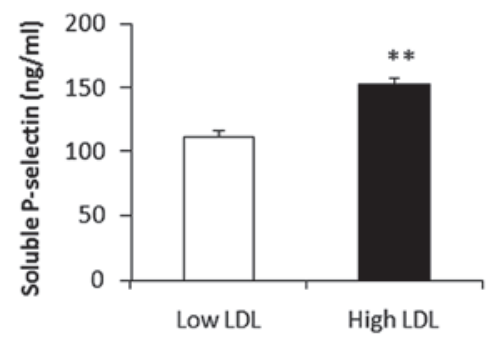

B

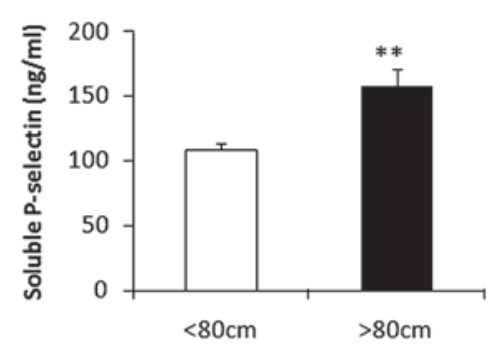

$\mathbf{D}$

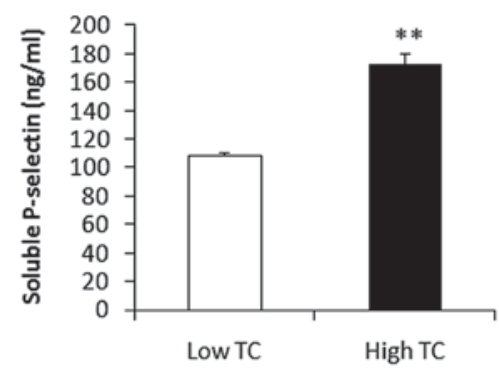

F

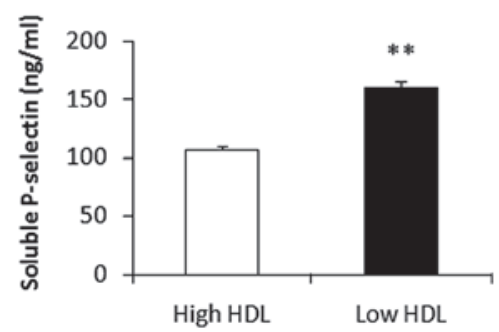

Figure 1. Soluble $\mathrm{P}$-selectin (sP-selectin) is increased in patients with obesity and hyperlipidemia. Plasma concentration of sP-selectin in (A) three body mass index (BMI) groups $(\mathrm{BMI}<25, \mathrm{n}=17 ; 25<\mathrm{BMI}<30, \mathrm{n}=22$; BMI $>30, \mathrm{n}=11),(\mathrm{B})$ two waist circumference $(\mathrm{WC})$ groups $(\mathrm{WC}<80 \mathrm{~cm}, \mathrm{n}=29$; WC $>80 \mathrm{~cm}, \mathrm{n}=21)$ and $(\mathrm{C})$ two pericardial adipose tissue (PCAT) area groups (PCAT $<160 \mathrm{~cm}^{2}, \mathrm{n}=22 ;$ PCAT $>160 \mathrm{~cm}^{2}, \mathrm{n}=28$ ), in all the controls, angina patients and acute myocardial infarction patients before percutaneous coronary intervention or thrombolysis therapy. Plasma concentration of soluble P-selectin in low and high (D) total cholesterol (TC), (E)low-densitylipoprotein(LDL)and(F)high-densitylipoprotein(HDL) groups(TC:low TC<5ng/ml,highTC $>5 \mathrm{ng} / \mathrm{ml}$;LDL:lowLDL $<3 \mathrm{ng} / \mathrm{ml}$,highLDL $>3 \mathrm{ng} / \mathrm{ml}$; HDL: low HDL $<1.05 \mathrm{ng} / \mathrm{ml}$; high HDL $>1.05 \mathrm{ng} / \mathrm{ml})$. ${ }^{*} \mathrm{P}<0.05$ and ${ }^{* *} \mathrm{P}<0.01$, compared with the white bar; data are presented as the mean \pm SEM. 

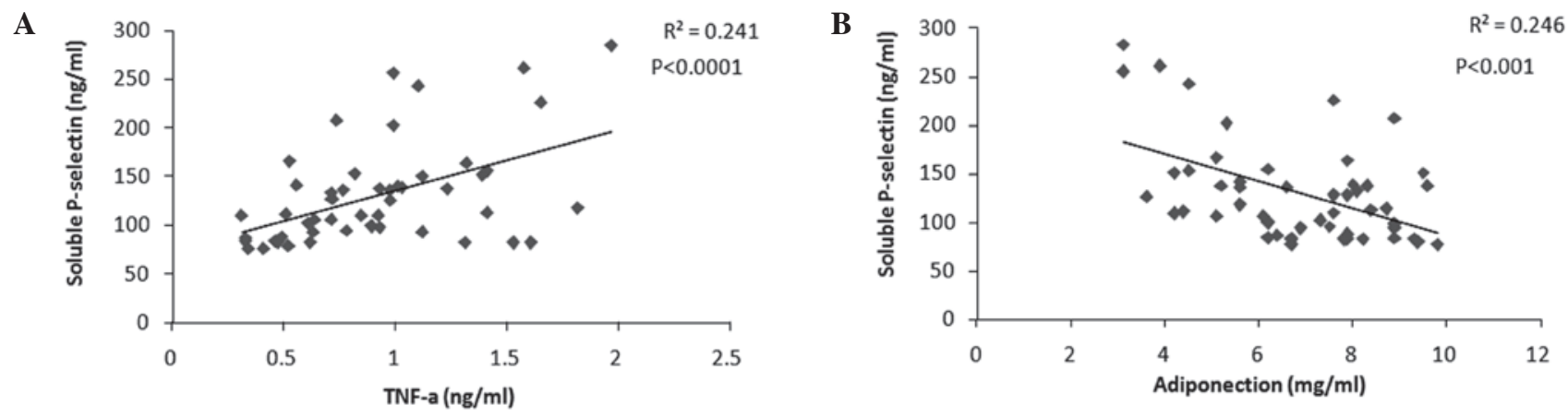

Figure 2. Soluble P-selectin (sP-selectin) is correlated with systemic inflammation. (A) Correlation between plasma concentration of sP-selectin and plasma tumor necrosis factor (TNF)- $\alpha$ in all cohorts. (B) Correlation between plasma concentration of sP-selectin and plasma adiponectin in all cohorts (n=50).

$\mathbf{A}$

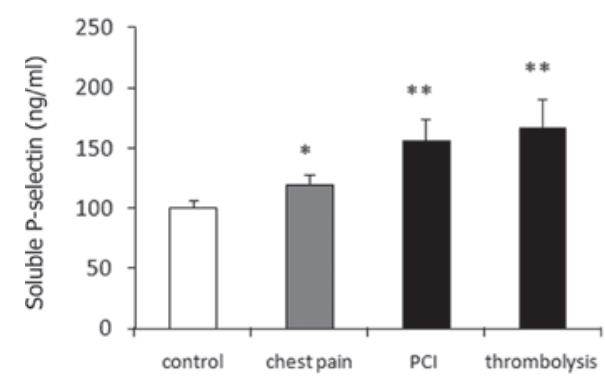

C

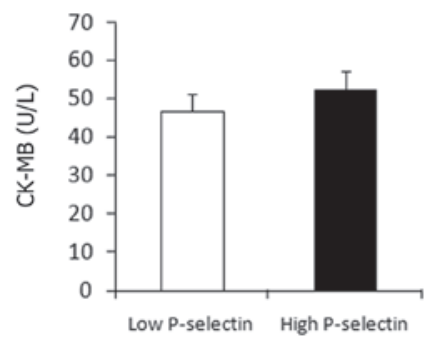

B

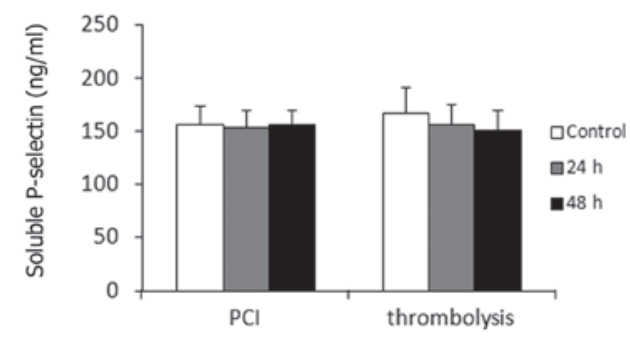

D

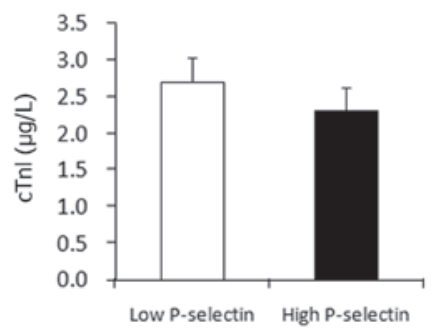

Figure 3. Soluble P-selectin (sP-selectin) is correlated with acute myocardial infarction (AMI) onset, but not AMI severity. (A) sP-selectin concentration in four groups [control, $\mathrm{n}=15$; chest pain, $\mathrm{n}=15$; percutaneous coronary intervention (PCI), $\mathrm{n}=10$; thrombolysis, $\mathrm{n}=10$ ]. (B) Change in sP-selectin concentration 24 and $48 \mathrm{~h}$ after PCI or thrombolysis treatment. Concentration of two AMI markers, (C) creatine kinase-MB (CK-MB) and (D) cardiac troponin I (cTnl) in the low sP-selectin $(<70 \mathrm{ng} / \mathrm{ml} ; \mathrm{n}=10)$ and high sP-selectin $(>70 \mathrm{ng} / \mathrm{ml} ; \mathrm{n}=10)$ groups before $\mathrm{PCI}$ and thrombolysis treatment. ${ }^{*} \mathrm{P}<0.05$ and ${ }^{* *} \mathrm{P}<0.01$, compared with the white bar; data are presented as the mean \pm SEM.

Obesity may lead to hyperlipidemia, which is a high risk factor for myocardial infarction, so next we confirmed the correlation between sP-selectin and hyperlipidemia. We revealed that $\mathrm{sP}$-selectin levels were significantly higher in high TC $(6.37 \pm 0.24 \mathrm{mmol} / \mathrm{l})$ patients than in low TC $(4.18 \pm 0.11 \mathrm{mmol} / \mathrm{l})$ patients $(173 \pm 15$ vs. $109 \pm 5 \mathrm{ng} / \mathrm{ml}$; Fig. 1D), sP-selectin levels were significantly higher in high LDL $(3.83 \pm 0.12 \mathrm{mmol} / \mathrm{l})$ patients than in low LDL $(2.12 \pm 0.16 \mathrm{mmol} / \mathrm{l})$ patients $(153 \pm 10 \mathrm{vs} .113 \pm 9 \mathrm{ng} / \mathrm{ml}$; Fig. 1E), and sP-selectin levels were significantly higher in low HDL $(0.94 \pm 0.04 \mathrm{mmol} / \mathrm{l})$ patients than in high HDL $(1.49 \pm 0.05 \mathrm{mmol} / \mathrm{l})$ patients $(160 \pm 12$ vs. $107 \pm 6 \mathrm{ng} / \mathrm{ml}$; Fig. 1F). In conclusion, sP-selectin levels were significantly higher in individuals with obesity (abdominal and pericardial obesity) and hyperlipidemia.

$s P$-selectin is positively correlated with chronic inflammation. As we have confirmed that sP-selectin is increased in individuals with obesity and hyperlipidemia, and it has been reported that obesity and hyperlipidemia lead to a chronic inflammation state (21), we hypothesized that sP-selectin was also correlated with chronic inflammation. To confirm this, we measured the serum TNF- $\alpha$ and adiponectin levels. It was demonstrated that $\mathrm{SP}$-selectin was positively correlated with serum TNF- $\alpha$ (Fig. 2A) and negatively correlated with serum adiponectin (Fig. 2B).

The above results suggest that chronic inflammation associated with obesity and hyperlipidemia may induce an increase in sP-selectin levels. The increased sP-selectin may play a significant role in certain inflammation-induced diseases.

$s P$-selectin is correlated with AMI onset but not AMI severity. Obesity, hyperlipidemia and chronic inflammation have been shown to be risk factors of AMI (17). It has been reported that sP-selectin may lead to cardiovascular diseases (4). Therefore, we firstly confirmed that $\mathrm{sP}$-selectin was significantly higher in PCI or thrombolysis therapy patients than in chest pain or control patients (Fig. 3A). Next, we considered whether PCI or 
A
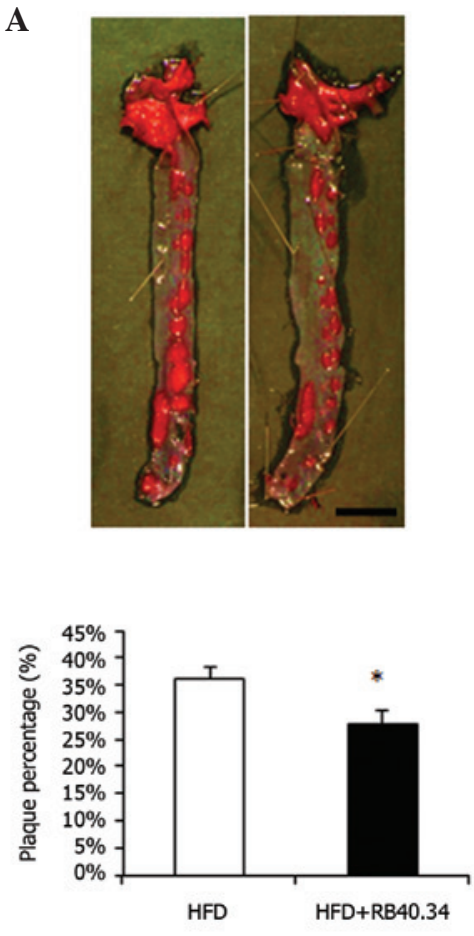

C
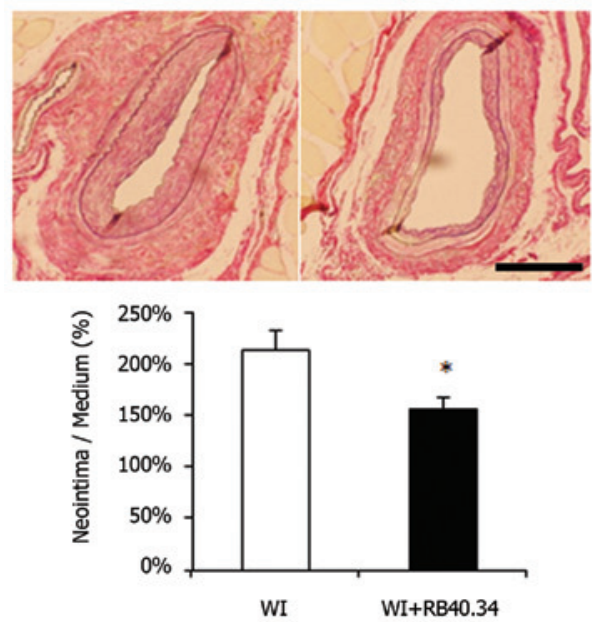

B
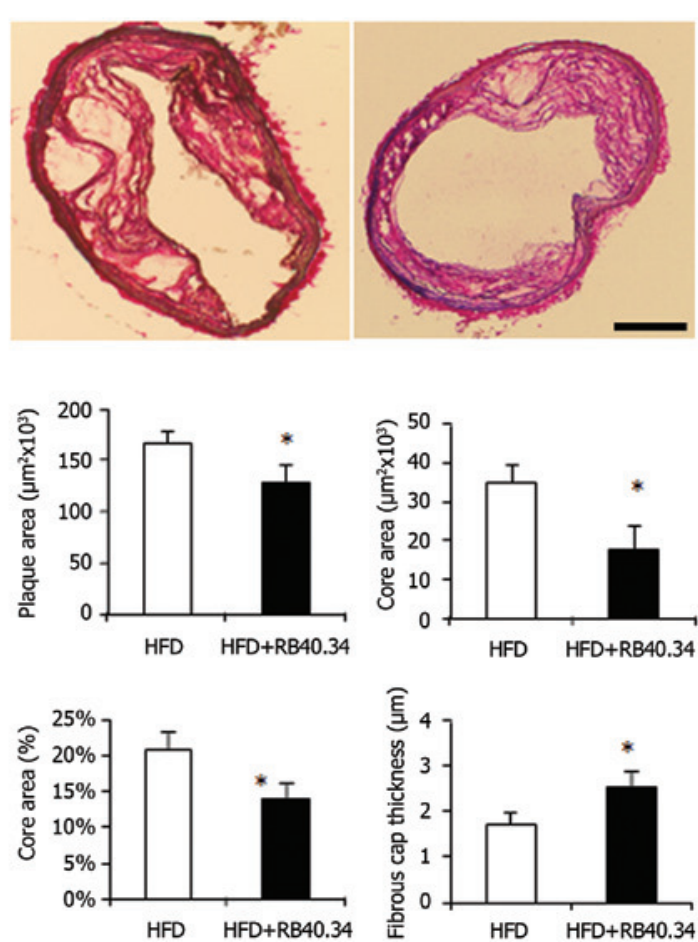

D
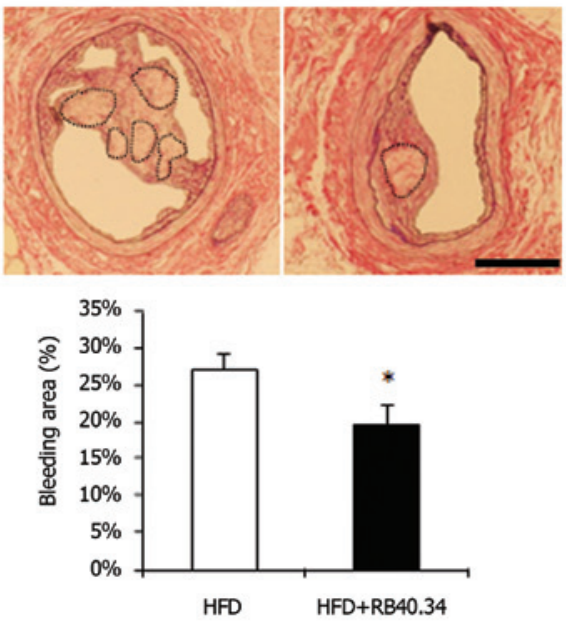

Figure 4. Soluble P-selectin induces arteriosclerotic plaque formation and its rupture, neointimal formation and neointimal bleeding. (A) Image of arteriosclerotic plaque formation in $\mathrm{ApoE}^{-/-}$mice fed a high-fat diet (HFD; left) and with RB40.34 administration (right) with quantification of the plaque area and aortic area ratio (scale bar, $0.5 \mathrm{~cm}$ ). (B) Image of carotid arterial arteriosclerotic plaque rupture with quantification of plaque stability indices (scale bar, $100 \mathrm{~mm}$ ) (C) Image of neointimal formation in wild-type mice following wire injury (left) and RB40.34 administration (right) with quantification of neointimal/medium ratio. (D) Image of neointimal bleeding in HFD wild-type mice (left; $n=14$ ) and in the HFD+RB40.34 administration group (right; $\mathrm{n}=15$ ) with quantification of the bleeding area/neointimal area. Magnification, $\mathrm{x} 20 .{ }^{*} \mathrm{P}<0.05$; data are presented as the mean $\pm \mathrm{SEM}$.

thrombolysis could decrease the sP-selectin level. To investigate this we measured the sP-selectin levels 24 and $48 \mathrm{~h}$ after PCI or thrombolysis treatment, and compared it with those before treatment. sP-selectin was not found to be affected by PCI or thrombolysis (Fig. 3B).

It was known that sP-selectin could induce AMI onset, but it was unclear whether sP-selectin affected AMI severity. In order to elucidate this, we divided all the PCI and thrombolysis therapy patients into two groups according to their sP-selectin levels: A low sP-selectin $(55 \pm 4 \mathrm{ng} / \mathrm{ml})$ group and a high sP-selectin $(107 \pm 8 \mathrm{ng} / \mathrm{ml})$ group. Subsequently, we compared the CK-MB and cTnl levels prior to any treatment. We demon- strated that in all AMI patients, there was no difference in CK-MB and cTnl levels between the low sP-selectin and high sP-selectin groups (Fig. 3C and D). These results demonstrate that $\mathrm{SP}$-selectin did not affect AMI severity.

sP-selectin induces atherosclerotic plaque and rupture, and neointimal formation and bleeding. We have confirmed that sP-selectin induces AMI onset but does not affect AMI severity. AMI is the result of coronary atherosclerosis and its rupture; hence, we next investigated whether sP-selectin was capable of inducing atherosclerotic plaque formation. In order to investigate this, $\mathrm{ApoE}^{-/-}$mice were used to create the atherosclerotic 
plaque model. Next, we administered RB40.43, a blocking monoclonal antibody of sP-selectin, to block sP-selectin. As a result, the RB40.43 administration group mice were found to exhibit decreased atherosclerotic plaque formation (Fig. 4A). AMI was caused by unstable plaque and rupture of plaque. Notably, inhibition of sP-selectin also suppressed necrotic core formation, and thus enhanced fibrous cap thickness (Fig. 4B). These results indicated that $\mathrm{sP}$-selectin could induce atherosclerotic plaque formation and its rupture.

Neointimal formation has been reported as the endothelial initial remodeling of atherosclerosis, and prevention of injury-induced neointimal formation also reduces spontaneous atherosclerosis (23). For this reason, we considered whether sP-selectin was capable of inducing neointimal formation. To investigate this, normal wild-type mice were used to create a wire-injured neointimal formation model, and RB40.43 was administered. As a result, the mice exhibited weak neointimal formation compared with the control group (Fig. 4C). As neointimal hyperplasia also exhibited neointimal bleeding in certain model mice (16), we used a high-fat diet (HFD) to induce potential neointimal bleeding. We revealed that, in the HFD group, half of the mice exhibited neointimal bleeding; however, in the RB40.34 administration group, only $27 \%$ of mice exhibited neointimal bleeding (data not shown). Among the bleeding mice, the RB40.34 administration group exhibited a smaller degree of bleeding compared with the HFD group, and we also demonstrated that inhibition of sP-selectin by RB40.34 reduced the bleeding area (Fig. 4D). These results indicate that $\mathrm{sP}$-selectin induced neointimal formation and bleeding.

\section{Discussion}

P-selectin is a transmembrane protein present in the alpha granules of platelets and the Weibel-Palade bodies of endothelial cells. P-selectin expression in platelets and endothelial cells has been shown to be elevated in disorders associated with cardiovascular diseases (24). Animal models have demonstrated the significant roles of P-selectin in the process of these cardiovascular diseases. For example, increased P-selectin expression has been demonstrated in active atherosclerotic plaque; in contrast, animals lacking P-selectin have a decreased tendency to form atherosclerotic plaque. Increased levels of soluble P-selectin in the plasma have also been demonstrated in a variety of cardiovascular disorders, including coronary artery disease, hypertension and atrial fibrillation, with some correlation with prognosis [as reviewed in (4)]. However, it was only known that $\mathrm{SP}$-selectin was capable of inducing AMI, and not whether sP-selectin also affected severity in patients with existing AMI.

It is reported that fibrotic inactive plaque lacks sP-selectin expression (25), which indicated the possibility that a lack of sP-selectin would lead to relatively stable plaque, a relatively safe state against the AMI onset for atherosclerosis patients. Although AMI onset was positively correlated with the formation of atherosclerotic plaque, in which patients the sP-selectin levels were elevated, not all patients with atherosclerotic plaque will progress to AMI. Although unstable plaque is not synonymous with AMI (26), unstable plaque notably led to more patients with AMI (27). Therefore, we assumed that sP-selectin was a risk factor in promoting unstable plaque formation, and furthermore inducing the onset of AMI. However, in individuals already suffering AMI, AMI severity has become the most significant index for prognosis (28). Therefore, in this study, we investigated the role of sP-selectin in the onset and severity of AMI.

Obesity, hyperlipidemia and systemic inflammation are reported as risk factors for AMI and other cardiovascular diseases $(29,30)$. Hence, in the present study, we firstly confirmed that sP-selectin was increased in obesity and hyperlipidemia patients, and also correlated with systemic inflammation (Figs. 1 and 2). These results were consistent with those of previous studies (31-33). We additionally reported that $\mathrm{sP}$-selectin levels were increased in patients with a higher PCAT area. However, the reason for the association between sP-selectin and PCAT area remains unknown. Notably, sP-selectin is also increased in patients with type 1 and type 2 diabetes mellitus $(34,35)$, which also has systemic chronic inflammatory status, so this evidence also supports the inflammation-promotion role of sP-selectin. It is a limitation of the present study that the diabetes mellitus background of patients was not investigated. The abovementioned studies and our data indicate that obesity, hyperlipidemia and systemic chronic inflammation are the basic risk factors to induce sP-selectin.

The present study also confirmed that sP-selectin was increased in AMI patients (Fig. 3A), which was consistent with a previous study (36). Hence, methods of reducing the sP-selectin level have become a significant means of preventing AMI. PCI and thrombolysis are extremely effective therapies; however, neither PCI nor thrombolysis were capable of reducing $\mathrm{sP}$-selectin (Fig. 3B), and sP-selectin did not affect the severity of AMI before patients accepted PCI or thrombolysis (Fig. 3C). Therefore, it was concluded that sP-selectin only induced AMI onset, but did not affect its severity. The plasma level of sP-selectin was increased in patients with unstable angina(Fig. 3A). Transient coronary arterial spasm could activate coronary arterial endothelium cells, and also lead to a high inflammatory status followed by transient myocardial ischemia. Activated coronary arterial endothelium cells and inflammation induce an increase in sP-selectin (37). However, although PCI and thrombolysis reduce systemic inflammation through enlargement of the narrow coronary arterial lumen and breakdown of apolipoprotein by thrombolysis (38), sP-selectin was not reduced. It is possible that in PCI patients, although stenosis was improved and systemic inflammation was decreased, the catheter would injure the coronary arterial endothelium cells, which would lead to a potential increase in inflammation. Alternatively, the reduction in sP-selectin may take a relatively long time following PCI or thrombolysis treatments, but in the present study we only investigated up to $48 \mathrm{~h}$ post-treatment. AMI severity depends on the location of coronary arterial stenosis and the hospitalization time. In the present study, we investigated $15 \mathrm{PCI}$ and 15 thrombolysis patients, and the hospitalization time for the two groups of patients was almost the same (data not shown). Therefore, sP-selectin has no correlation with the location of coronary arterial stenosis.

As superimposed luminal thrombus and rupture of coronary atherosclerosis are main causes of AMI, we believed atherosclerosis plaque formation to be a further cause of AMI, induced by sP-selectin. Since neointimal formation was thought to be 
the initial pathological change of atherosclerosis, we hypothesized that $\mathrm{sP}$-selectin could induce atherosclerosis plaque and neointimal formation (Fig. 4A and C). Increased plaque is an increased risk factor for coronary artery stenosis, and once the stenosis reaches $70 \%$, obstructive coronary artery disease may be defined. Once patients are diagnosed with obstructive coronary artery disease, the stability of the plaque is the critical issue to address, as unstable plaque leads directly to AMI. In the present study, we demonstrated that sP-selectin inhibitor not only reduced plaque rupture but also decreased the neointimal bleeding incidence rate and the bleeding area (Fig. 4B and D).

In conclusion, in the current study, we demonstrated that an excess of sP-selectin, induced by systemic inflammation and hyperlipidemia, leads to neointimal hyperplasia and atherosclerosis plaque formation with unstable neointimal bleeding and plaque rupture, and then leads to the onset of AMI. However, in individuals already suffering AMI, a further increase of sP-selectin may have no effect on severity.

\section{References}

1. Geng JG, Chen M and Chou KC: P-selectin cell adhesion molecule in inflammation, thrombosis, cancer growth and metastasis. Curr Med Chem 11: 2153-2160, 2004.

2. Polek A, Sobiczewski W and Matowicka-Karna J: P-selectin and its role in some diseases. Postepy Hig Med Dosw (Online) 63 : 465-470, 2009.

3. Yang J, Furie BC and Furie B: The biology of P-selectin glycoprotein ligand-1: its role as a selectin counterreceptor in leukocyte-endothelial and leukocyte-platelet interaction. Thromb Haemost 81: 1-7, 1999.

4. Blann AD, Nadar SK and Lip GY: The adhesion molecule P-selectin and cardiovascular disease. Eur Heart J 24: 2166-2179, 2003.

5. Ramacciotti E, Blackburn S, Hawley AE, et al: Evaluation of soluble P-selectin as a marker for the diagnosis of deep venous thrombosis. Clin Appl Thromb Hemost 17: 425-431, 2011.

6. Woollard KJ and Chin-Dusting J: P-selectin antagonism in inflammatory disease. Curr Pharm Des 16: 4113-4118, 2010.

7. Burke AP and Virmani R: Pathophysiology of acute myocardial infarction. Med Clin North Am 91: 553-572, 2007.

8. Woollard KJ and Chin-Dusting J: Therapeutic targeting of p-selectin in atherosclerosis. Inflamm Allergy Drug Targets 6: 69-74, 2007.

9. Aversano T, Aversano LT, Passamani E, et al: Thrombolytic therapy vs primary percutaneous coronary intervention for myocardial infarction in patients presenting to hospitals without on-site cardiac surgery: a randomized controlled trial. JAMA 287: 1943-1951, 2002.

10. Ferroni P, Basili S, Martini F, et al: Soluble P-selectin as a marker of platelet hyperactivity in patients with chronic obstructive pulmonary disease. J Investig Med 48: 21-27, 2000.

11. Ertenli I, Kiraz S, Arici M, et al: P-selectin as a circulating molecular marker in rheumatoid arthritis with thrombocytosis. J Rheumatol 25: 1054-1058, 1998.

12. Tamagawa-Mineoka R, Katoh N, Ueda E, Masuda K and Kishimoto S: Platelet-derived microparticles and soluble P-selectin as platelet activation markers in patients with atopic dermatitis. Clin Immunol 131: 495-500, 2009.

13. Konishi M, Sugiyama S, Sugamura K, et al: Association of pericardial fat accumulation rather than abdominal obesity with coronary atherosclerotic plaque formation in patients with suspected coronary artery disease. Atherosclerosis 209: 573-578, 2010.

14. Zhou J, Lhotak S, Hilditch BA and Austin RC: Activation of the unfolded protein response occurs at all stages of atherosclerotic lesion development in apolipoprotein E-deficient mice. Circulation 111: 1814-1821, 2005.

15. Johnson JL and Jackson CL: Atherosclerotic plaque rupture in the apolipoprotein E knockout mouse. Atherosclerosis 154: 399-406, 2001.

16. Sata M, Maejima Y, Adachi F, et al: A mouse model of vascular injury that induces rapid onset of medial cell apoptosis followed by reproducible neointimal hyperplasia. J Mol Cell Cardiol 32: 2097-2104, 2000.
17. Phillips JW, Barringhaus KG, Sanders JM, et al: Single injection of P-selectin or P-selectin glycoprotein ligand-1 monoclonal antibody blocks neointima formation after arterial injury in apolipoprotein E-deficient mice. Circulation 107: 2244-2249, 2003.

18. Lin WY, Lee LT, Chen CY, et al: Optimal cut-off values for obesity: using simple anthropometric indices to predict cardiovascular risk factors in Taiwan. Int J Obes Relat Metab Disord 26: 1232-1238, 2002.

19. Guo L, Ning W, Tan Z, Gong Z and Li X: Mechanism of matrix metalloproteinase axis-induced neointimal growth. J Mol Cell Cardiol 66: 116-125, 2013.

20. Konishi M, Sugiyama S, Sato Y, et al: Pericardial fat inflammation correlates with coronary artery disease. Atherosclerosis 213: 649-655, 2010.

21. Hamminga EA, van der Lely AJ, Neumann HA and Thio HB: Chronic inflammation in psoriasis and obesity: implications for therapy. Med Hypotheses 67: 768-773, 2006.

22. Hackam DG and Anand SS: Emerging risk factors for atherosclerotic vascular disease: a critical review of the evidence. JAMA 290: 932-940, 2003.

23. Quarck R, De Geest B, Stengel D, et al: Adenovirus-mediated gene transfer of human platelet-activating factor-acetylhydrolase prevents injury-induced neointima formation and reduces spontaneous atherosclerosis in apolipoprotein E-deficient mice. Circulation 103: 2495-2500, 2001.

24. Merten $\mathrm{M}$ and Thiagarajan P: P-selectin in arterial thrombosis. Z Kardiol 93: 855-863, 2004.

25. Hong MK, Mintz GS, Lee CW, et al: Comparison of coronary plaque rupture between stable angina and acute myocardial infarction: a three-vessel intravascular ultrasound study in 235 patients. Circulation 110: 928-933, 2004.

26. Lee SG, Lee CW, Hong MK, Kim JJ, Park SW and Park SJ: Change of multiple complex coronary plaques in patients with acute myocardial infarction: a study with coronary angiography. Am Heart J 147: 281-286, 2004.

27. Finn AV, Nakano M, Narula J, Kolodgie FD and Virmani R: Concept of vulnerable/unstable plaque. Arterioscler Thromb Vasc Biol 30: 1282-1292, 2010.

28. Claeys MJ, Bosmans J, Veenstra L, Jorens P, De Raedt H and Vrints CJ: Determinants and prognostic implications of persistent ST-segment elevation after primary angioplasty for acute myocardial infarction: importance of microvascular reperfusion injury on clinical outcome. Circulation 99: 1972-1977, 1999.

29. Triant VA, Lee H, Hadigan C and Grinspoon SK: Increased acute myocardial infarction rates and cardiovascular risk factors among patients with human immunodeficiency virus disease. J Clin Endocrinol Metab 92: 2506-2512, 2007.

30. Rosengren A, Wallentin L, Simoons M, et al: Cardiovascular risk factors and clinical presentation in acute coronary syndromes. Heart 91: 1141-1147, 2005.

31. Kvasnicka T, Kvasnicka J, Ceska R, Grauova B and Vrablik M: Increasing plasma levels of soluble cell adhesion molecules (sE-selectin, sP-selectin and sICAM-1) in overweight adults with combined hyperlipidemia. Sb Lek 102: 473-477, 2001.

32. De Pergola G, Pannacciulli N, Coviello M, et al: sP-selectin plasma levels in obesity: association with insulin resistance and related metabolic and prothrombotic factors. Nutr Metab Cardiovasc Dis 18: 227-232, 2008.

33. Larsson PT, Hallerstam S, Rosfors S and Wallen NH: Circulating markers of inflammation are related to carotid artery atherosclerosis. Int Angiol 24: 43-51, 2005.

34. Yngen M, Ostenson CG, Hu H, Li N, Hjemdahl P and Wallen NH: Enhanced P-selectin expression and increased soluble CD40 Ligand in patients with Type 1 diabetes mellitus and microangiopathy: evidence for platelet hyperactivity and chronic inflammation. Diabetologia 47: 537-540, 2004.

35. Gokulakrishnan K, Deepa R, Mohan V and Gross MD: Soluble P-selectin and CD40L levels in subjects with prediabetes, diabetes mellitus, and metabolic syndrome - the Chennai Urban Rural Epidemiology Study. Metabolism 55: 237-242, 2006.

36. Xu DY, Zhao SP and Peng WP: Elevated plasma levels of soluble P-selectin in patients with acute myocardial infarction and unstable angina. An inverse link to lipoprotein(a). Int J Cardiol 64: 253-258, 1998.

37. Atalar E, Aytemir K, Haznedaroglu I, et al: Increased plasma levels of soluble selectins in patients with unstable angina. Int $\mathrm{J}$ Cardiol 78: 69-73, 2001.

38. Eberini I, Gianazza E, Breghi L, et al: Apolipoprotein A-I breakdown is induced by thrombolysis in coronary patients. Ann Med 39: 306-311, 2007. 